\title{
Lateral Vehicle Control for Semi-Autonomous Valet Parking with Consideration of Actuator Dynamics
}

\author{
Dohyun Kim, Bongsob Song*
}

Department of Mechanical En gin eering,Ajou University, Suwon, 443-749, Korea

\begin{abstract}
In this paper, the lateral control algorith $m$ for semi-autonomous valet parking is presented and its feasibility is demonstrated via field driving tests. With the assumptions of low speed driving and s mall slip angle, a vehicle model with kinematic constraints of a steering actuator is proposed todesign the lateral controller. A model-based nonlinear control technique called dyna mic surface control is applied to developa lateral control law for forward driving and backward parallel parking maneuvers. Furthermore, the previewcontrol and filteringtechniquesare incorporated in the lateral controller to improve the tracking performance. Since there is measurement noiseregarding position and yaw angle and model uncertainty, it is necessary for the proposed lateral controller to be robust enough to compensate for noise and disturbance. Finally the performance of the lateral controller is validated experimentally via field tests as well as simulations.
\end{abstract}

Keywo rds Lateral Control, Semi-autonomous Valet Parking, Parallel Parking, Nonlinear Control

\section{Introduction}

The growing attention has been paid to a parking assistance system (PAS) to provide more safety and convenience to a driver. The PASallows the driver to park a car without manual steering control and has been commercializedrecently by many automakers[1]. Furthermore, the advanced parking assistance system (APAS) (or called self-parking), which makes a vehicle parked autonomously, has been developed[2, 3].For instance, autonomous driving including autonomous valet parking (A VP) was demonstrated in the Urban Challengein 2007, which is an autonomous vehicle competition[4, 5]. Whereas it succeeded in showing the feasibility of autonomous drivingwith many additional sensors such as radars, lidars, cameras, and ultrasonic sensors to recognize lane, obstacles, and a parking lot, it is interesting to remark that the detection range to identify an available parking lot in a public parking structure is still limited.

Another approach is to develop an intelligent parking infrastructure whichprovides usefully driving information such as position of the vehicle, obstacles and location of anavailable parking space to either a driver or a vehicle wirelessly via vehicle to infrastructure (V2I) communication. Since a parking guidance system to identify an optimal parking lot and guide the route to the driver has been already developed[6], the development of

* Corresponding author:

bsong@ajou.ac.kr (Bongsob Song)

Published online at http://journal.sapub.org/control

Copyright (C) 2012 Scientific \& Academic Publishing. All Rights Reserved the intelligent parking infrastructure is feasible if the positions of all vehicles in the parking structure can be measured or estimated.

One possible approach to obtain this information is to use a set of infrastructure sensors such as lidars and cameras, which are implemented in the parking structure, not on the vehicle[7]. That is, the infrastructure sensors detect all vehicles within their detection range, and positions of vehicles are estimated by combining all measurements of sensors and location of sensors centrally in an intelligent parking server.

In this paper, a semi-autonomous valet parking (SA VP) system with the cooperation of the intelligent parking infrastructure is considered under the assumption that a driver is in a vehicle and the velocity is controlled manually. Only steering control is performed automatically for forward driving to a parking lot and backward parking maneuvers. Furthermore, it is assumed that an optimal parking lot is identified and the corresponding route, i.e., a set of waypoints, is planned by the intelligent parking server, and the corresponding information is sent to the vehicle via V2I communication.

Among many challenging problems for SA VP in the area of perception, planning, communication, and control, a lateral control problem is only focused in this paper. More specifically speaking, the challenging control problems to be solved in this paper are summarized as follows:

-Both measurement noise (especially in position and heading angle) and model uncertainty are considered.

-Various driving maneuvers (e.g., forward driving, temporary stop, and backward parking) need to be conducted in a unified framework of lateral control. 
-The driving performance should reflect the behavior of a human driver. Thus, the movement of steering is slow enough to satisfy kinematic constraints of a steering actuator.

\section{Driving Scenario and Hardware}

As shown in Fig. 1, it is assumed that the driving scenario for SAVP includes a series of driving maneuvers, i.e., forward driving on a straight and curved road (see a waypoint $A$ to $B$ in Fig. 1), temporary stop (at $B$ in the figure), backward parallel parking ( $B$ to $C$ in the figure), and complete stop at the waypoint $C$. Moreover, it is assumed that an available parking location is determined by an SA VP server which monitors all vehicles in a dedicated intelligent parking infrastructure. Then, the corresponding waypoints (refer to the circles in Fig. 1) are delivered to the vehicle controller via V2I communications.

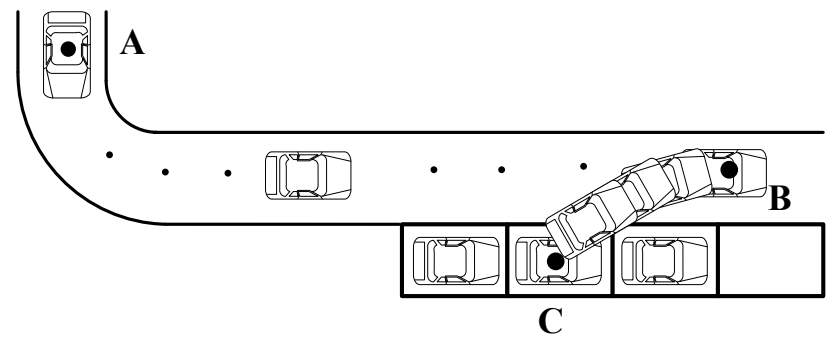

Figure 1. Driving Scenario for SAVP

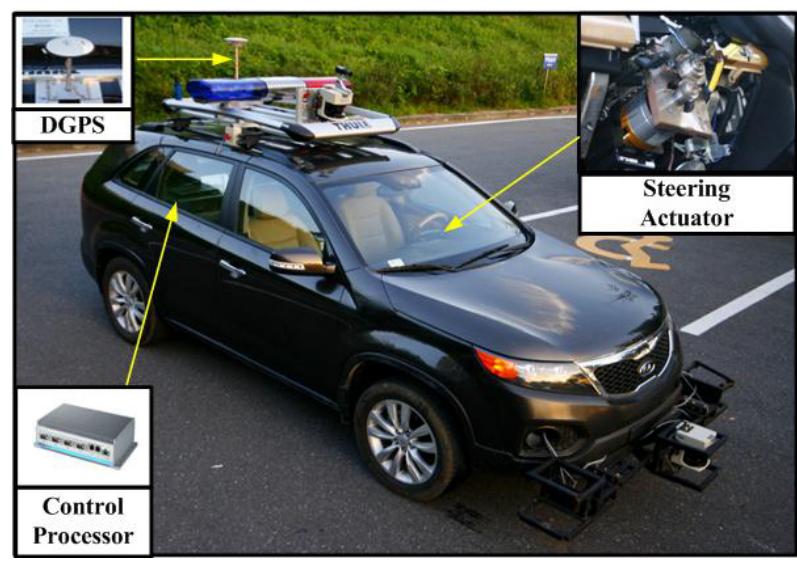

Figure 2. Hardware Layout of Test Vehicle

To conduct the given driving maneuvers for SA VP, a test vehicle shown in Fig. 2 has the following capabilities:

-The steering wheel angle can be controlled by external commands determined from the vehicle controller. In fact, it is feasible if a motor drive power steering system (MDPS) which is already commercialized is available.

-Both position and heading angle of the vehicle with measurement noise are provided. It is noted that a DGPS is used for experimental validation of the proposed lateral controller as shown in Fig.2 although it will be replaced by infrastructure sensors in the near future.

-There is an electronic control unit (ECU) or processor to compute a steering angle command based on information via V2I communications.
- Gear engagement and wheel speed are measured by in-vehicle sensors and the information is sent to ECU via controller area network (CAN).

\section{Lateral Control}

The lateral controller to provide the desired steering wheel angle for SA VP needs both trajectory for different driving maneuvers and control laws. A kinematic vehicle model subject to kinematic constraints of the steering actuator is proposed and the model-based nonlinear control technique is applied for the controller design.

\subsection{Trajectory Generation}

To perform different driving maneuvers such as forward driving and backward parallel parkingas illustrated in Fig. 1, the corresponding trajectory is necessary for the lateral controller. Three approaches for the trajectory generation have been found mainly in the literature: a combination of lines and circles[8,9], curve fitting[10], and clothoids[11]. In this section, both the combination of circles and curve fitting techniques are used for trajectory generation.

In the case of forward driving, the trajectory generation is interpreted as curve interpolation between two given waypoints. Suppose two waypoints are given as $p_{0}$ (or a point $A$ ) and $p_{3}$ (or a point $B$ ) as seen in Fig. 3. Ifa cubic Bezier curve interpolation approach is appliedwith two additional control points, $p_{1}$ and $p_{2}$, the trajectory for the forward driving isdetermined as follows:

$$
P_{d}(k)=a u_{k}^{3}+b u_{k}^{2}+c u_{k}+p_{0}
$$

where $P_{d}(k)=\left[\begin{array}{ll}x_{d}(k) & y_{d}(k)\end{array}\right]^{T} \in \mathfrak{R}^{2}$ is the desired trajectory (or a set of reference points) between two waypoints with respect to a local coordinate originated at $p_{0}, u_{k} \in\left[\begin{array}{ll}0 & 1\end{array}\right]$ is equally spacing for $k=1, \ldots, n$, and the vectors $a, b$, and $c$ are defined as

$$
\begin{aligned}
& c=3\left(p_{1}-p_{0}\right) \in \mathfrak{R}^{2}, \quad b=3\left(p_{2}-p_{1}\right)-c \in \mathfrak{R}^{2}, \\
& a=p_{3}-p_{0}-b-c \in \mathfrak{R}^{2}
\end{aligned}
$$

The shape and smoothness of the trajectory in (1) depends on the selection of two control point points(see $p_{1}$ and $p_{2}$ in Fig. 3). The control points are written in the coordinate from as follows:

$$
\begin{aligned}
& p_{1}=p_{0}+l \angle \phi_{1}=p_{0}+l \angle \psi, \\
& p_{2}=p_{3}-l \angle \phi_{2}
\end{aligned}
$$

wherel is a constant which determines the smoothness of trajectory, $\psi$ is the yaw angle of the vehicle, and $\phi_{2}$ is the angle between $p_{3}$ and next waypoint. However, it is noted that $\phi_{2}$ is a predefined angle when the vehicle reaches the waypoint where the parking maneuver begins (refer to a waypoint $B$ in Fig. 3).

For the backward parallel parking, one of the simplest trajectory generation techniques is to use two circles based on Ackermann steering geometry of the vehicle[6]. When the vehicle arrives at an available parking location, i.e., near 
a waypoint Bin Fig. 3, it is assumed that a parking end point, i.e., a waypoint $C$ in Fig. 3 , is given via V2I communication.To generate two circles for parallel parking, an intersection point of two circles, $Q$ in Fig. 3, is first calculated under the assumption that two circles with the same radius are used. Since a circle is uniquely determined when two points on the circle are known, a center and radius of the circle passing a current position near a waypoint $B$ and $Q$ can be determined as follows:

$$
\begin{aligned}
S_{x} & =B_{x} \\
S_{y} & =\frac{1}{2\left(Q_{y}-B_{y}\right)}\left[\left(Q_{x}-S_{x}\right)^{2}-\left(B_{x}-S_{x}\right)^{2}-B_{y}^{2}+Q_{y}^{2}\right] \\
r & =\|Q-S\|=\|B-S\|
\end{aligned}
$$

Since the center of the second circle, $T$, can be obtained similarly, the trajectory for the parallel parking can be summarized as follows:

$$
\begin{cases}\left(x_{d}-S_{x}\right)^{2}+\left(y_{d}-S_{y}\right)^{2}=r^{2} & \text { if } \mathrm{x} \geq Q_{x} \\ \left(x_{d}-T_{x}\right)^{2}+\left(y_{d}-T_{y}\right)^{2}=r^{2} & \text { otherwise }\end{cases}
$$

where the subscript $d$ stands for thedesired trajectory, and thesubscripts $x$ and $y$ are the positions with respect to $x$ and $y$ directions respectively.

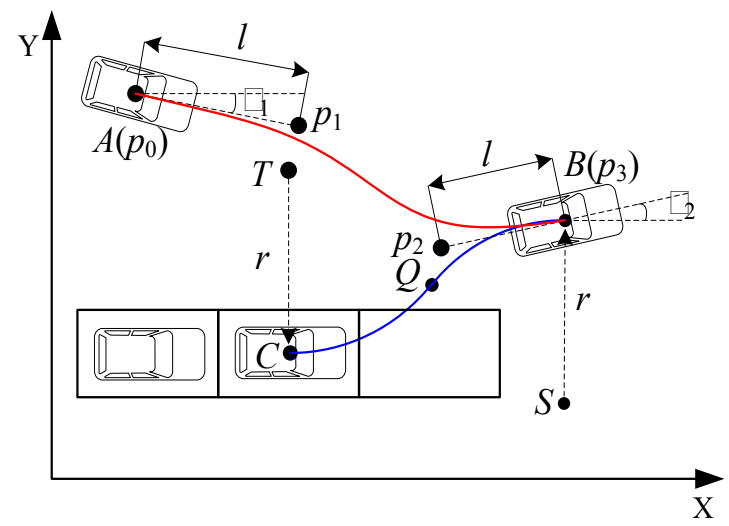

Figure 3. TrajectoryGeneration for SAVP

\subsection{Vehicle Model}

A bicycle model has been used widely for design of the lateral controller for highway driving[12]. It is assumed that all system parameters of tire stiffness are known and all wheel angles are small. However, a large amount of wheel angle is generated for parking and it is not easy to identify the system parameters for various types of vehicles.

For the SA VP, the vehicle is driving at low speed and thus sides lip angle is neglected in this study. Thus, the kinemat ic model can be used for both driving and parallel parking. The equation of motion is written as follows [13, 14]:

$$
\begin{aligned}
& \dot{x}=v \cos \psi \\
& \dot{y}=v \sin \psi \\
& \dot{\psi}=\frac{v}{L_{i}} \tan \delta
\end{aligned}
$$

where $x$ and $y$ are the longitudinal and lateral positions of the vehicle, $v$ is the vehicle speed, and $\delta$ is the steering angle. In addition, the subscript $i$ represents the driving maneuver, i.e., $f$ is forward driving and $b$ is backward driving. It is noted that the effective length of wheelbase, $L_{i}$, instead of the length of wheelbase $(L)$, is used with respect to driving maneuver. Furthermore, the kinemat ic constraints of a steering actuator are considered and they are written as

$$
|\delta| \leq \delta_{\max }, \quad|\dot{\delta}| \leq \omega_{\max }
$$

where $\delta_{\max }, \omega_{\max }$ are the maximum steering angle and angular velocity respectively.

Suppose a set of parameters in (4) and (5) are given as

$$
\begin{aligned}
L_{f} & =2.9(\mathrm{~m}), L_{b}=2.2(\mathrm{~m}), \\
\delta_{\max } & =34(\mathrm{deg})=0.5934(\mathrm{rad}), \text { and } \omega_{\max }=0.3(\mathrm{rad} / \mathrm{s}) .
\end{aligned}
$$

The time responses of the vehicle model in (4) and (5) are compared with experimental data using a test vehicle shown in Fig. 2. The vehicle is driven forward manually from 0 to about 75 second, stopped temporarily to changegear engagement for backward driving, and driven backward from about 78 to 110 second (see the first plot in Fig. 4). The corresponding steering angle is shown in the second plot of Fig. 4. Around 78 second, the maximum steering angle is required for backward parking and the maximum angular velocity of the steering angle is shown at that time. The angular velocity is approximated by use of difference of the steering angle of five samples as follows:

$$
\Delta \delta / T(k)=(\delta(k+2)-\delta(k-2)) / 5 T
$$

Where $T$ is a sampling time.

When the same velocity and steering angle satisfying the constraints in (5) are assigned, it is shown in the fourth and fifth plots of Fig. 4 that both responses of forward driving and backward parking are quite similar with the maximum error deviation of $0.11(\mathrm{rad})$ and $0.01(\mathrm{rad} / \mathrm{s})$ in terms of the yaw angle and rate respectively
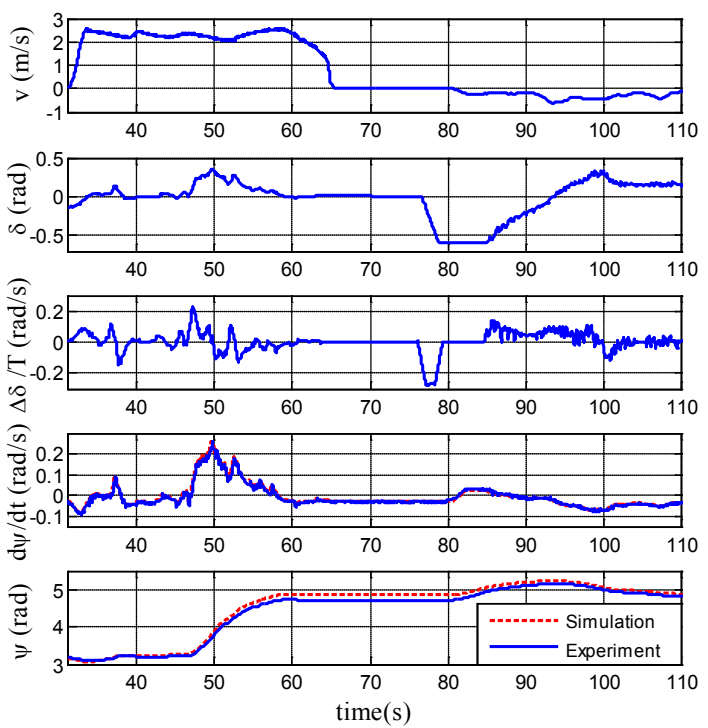

Figure 4. Comparisonbetween Vehicle Model and ExperimentalData 


\subsection{Lateral Control Law}

A model-based nonlinear control technique called dynamic surface control is applied to design alateral controllerat most of speed range[14, 15].First, the error surface is defined as follows (see also in Fig. 5)[14]:

$$
S_{i}=e_{y}+d_{i} e_{\psi}=e_{y}+d_{i}\left(\psi_{d}-\psi\right)
$$

where the lateral error $e_{y}$ is defined as

$$
e_{y}=\left\{\begin{array}{cc}
0 & \text { if } c=0 \\
-\operatorname{sign}(c)\left\|e_{d}\right\| & \text { otherwise, }
\end{array}\right.
$$

the shortest distance from the current position to the desired trajectory, $e_{d}$, is calculated as

$$
e_{d}=\left[\begin{array}{l}
x_{d}\left(k^{*}\right)-x \\
y_{d}\left(k^{*}\right)-y
\end{array}\right]
$$

Where $k^{*}$ is obtained iteratively by

$$
k^{*}=\arg \min _{k}\left\|P_{d}(k)-P\right\|=\arg \min _{k}\left\|\left[\begin{array}{l}
x_{d}(k) \\
y_{d}(k)
\end{array}\right]-\left[\begin{array}{l}
x \\
y
\end{array}\right]\right\|
$$

and $P_{d}$ comes from (1) for forward driving and (3) backward parking respectively. Furthermore, the sign of the lateral error in (7) is defined as follows:

$$
\begin{aligned}
c & =\operatorname{det}\left(\left[\begin{array}{c}
e_{p d}^{T} \\
-e_{d}^{T}
\end{array}\right]\right), \\
e_{p d} & =\left[\begin{array}{l}
x_{p d}-x_{d} \\
y_{p d}-y_{d}
\end{array}\right]=\left[\begin{array}{l}
x_{d}\left(k^{*}+m_{i}\right)-x_{d}\left(k^{*}\right) \\
y_{d}\left(k^{*}+m_{i}\right)-y_{d}\left(k^{*}\right)
\end{array}\right] .
\end{aligned}
$$

Where $\left[x_{p d} y_{p d}\right]^{\mathrm{T}}$ is the preview point and $m_{i}$ is a constant but two different values are assigned for forward driving and backward parking. When the vehicle is placed as in Fig. 5, $c$ is negative and the lateral error in (7) beco mes positive. Then, the positive (or counterclockwise) steering angle command will be determined by the lateral control law which will be discussed later. Furthermo re, $d_{i}$ and $\psi_{d}$ in (6) are defined as

$$
d_{i}=\left\|e_{p d}\right\|, \psi_{d}=\angle e_{p d}
$$

It is noted that the preview control idea is incorporated with DSC by considering both $d_{i}$ and $\psi_{d}$ in (6) [8].

After differentiating $S_{i}$ in (6) and combining it with (4), the time derivative of $S_{i}$ is

$$
\dot{S}_{i}=\dot{e}_{y}+d_{i}\left(\dot{\psi}_{d}-\dot{\psi}\right)=\dot{e}_{y}+d_{i} \dot{\psi}_{d}-\frac{d_{i} v}{L_{i}} \tan \delta .
$$

To make $S_{i}$ go to zero, let $\dot{S}_{i}=-K_{i} S_{i}$ where $K_{i}$ is acontroller gain. Then the desired steering angle is obtained as

$$
\begin{gathered}
\bar{g}=\tan \delta_{d e s}=\frac{L_{i}}{d_{i} v}\left(\dot{e}_{y}+d_{i} \dot{\psi}_{d}+K_{i} S_{i}\right) \\
\delta_{d e s}=\tan ^{-1}\left[\frac{L_{i}}{d_{i} v}\left(\dot{e}_{y}+d_{i} \dot{\psi}_{d}+K_{i} S_{i}\right)\right]
\end{gathered}
$$

Next, two possible cases which may make the control law in (10) violate the actuator constraints in (5) a re considered.
First, the desired steering angle in (10) can be changed rapidly due to the assignment of a large controller ga in $K_{i}$ or inclusion of measurement noise. Then the actual steering angle cannot follow the desired steering angle. To solve this problem, the lateral controller is redesigned by introducing a first order low-pass filter. That is, if the desired steering wheel is calculated after passing through as follows:

$$
\tau_{2} \dot{g}_{d e s}+g_{d e s}=\bar{g}, \quad g_{d e s}(0)=\bar{g}(0)
$$

the desired steering angle is determined as

$$
\delta_{\text {des }}=\tan ^{-1}\left(g_{\text {des }}\right)
$$

It is remarked that $\bar{g}$ in (9) rather than $\delta_{\text {des }}$ in (10) is filtered and it allows us to analyze the stability more easily and clearly[15].

For the second case, since the velocity is controlled manually by a driver, it can be very low or even zero. In this case, the desired steering angle in (10) becomes greater than $\delta_{\text {max }}$, thus resulting in saturation of the actuator regardless of the lateral error or $S_{i}$ in (6). Thus, two control laws are switched depending on velocity, i.e., a proportional controller is used when the velocity is very low. Otherwise, the lateral control law in (12) is used. Finally, the modified lateral control law is summarized as follows:

$$
\delta_{d e s}=\left\{\begin{array}{cc}
\tan ^{-1}\left(g_{d e s}\right) & \text { if }|v| \geq \varepsilon \\
K_{p i} S_{i} & \text { otherwise }
\end{array}\right.
$$

where $K_{p i}$ is a proportional controller gain and $\varepsilon$ is a velocity threshold.

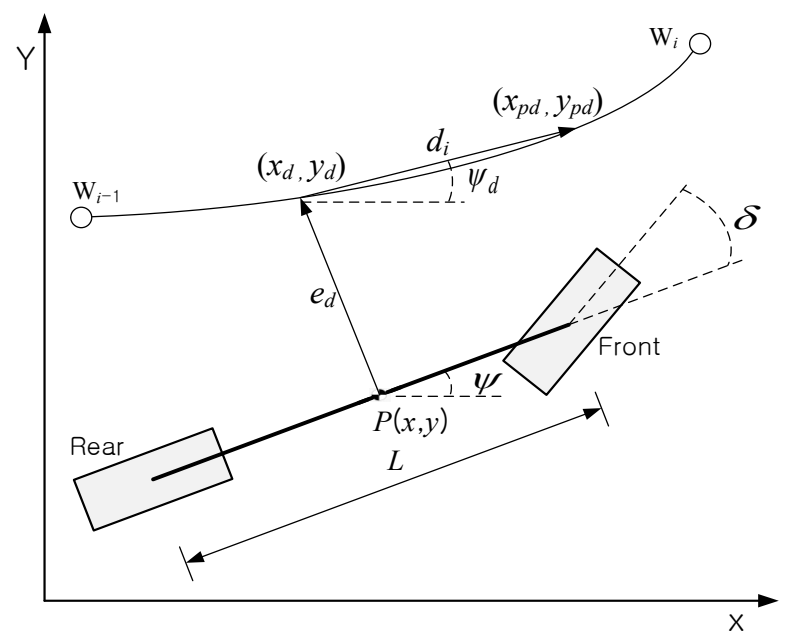

Figure 5. Definitions of Lat eral Error and Heading Angle Error

\section{Validation Results}

Suppose 21 waypoints are given as shown in Fig 6. The forward driving is required from the first to $20^{\text {th }}$ waypoint ( $A$ to $B$ in the figure) and the backward parallel parking is asked from $20^{\text {th }}$ to $21^{\text {st }}$ waypoint ( $B$ to $C$ in the figure). The proposed lateral controller is validated via a vehicle simulator called CarSim[16] and field tests using a test vehicle shown in Fig. 2. 
First, the tracking performance of the lateral control law in (13) is validated via simulations with considerations of measurement noise and kinematic constraints of a steering actuator. Before evaluating the performance, it is shown in Fig 6 that trajectory for forward driving and backward parallel parking is generated appropriately. When measurement noise of position and yaw angle is not considered, the performance of the lateral controller in (10) is shown in Fig. 6 and 7. It is validated in Fig. 7 that two different driving maneuvers can be conducted by the single lateral controller with the maximum lateral error deviation of about $0.2(\mathrm{~m})$ (see the top plot in Fig. 7).

However, it is shown in the bottom plot of Fig 7 that the steering angle does not track the desired steering angle sometimes due to the kinematic constraint of the steering angular velocity. If the control law in (13) is used, it is shown in Fig. 8 that the desired steering angle does not violate the kinematic constraints much less than one in (10) without any performance degradation in the term of lateral error.

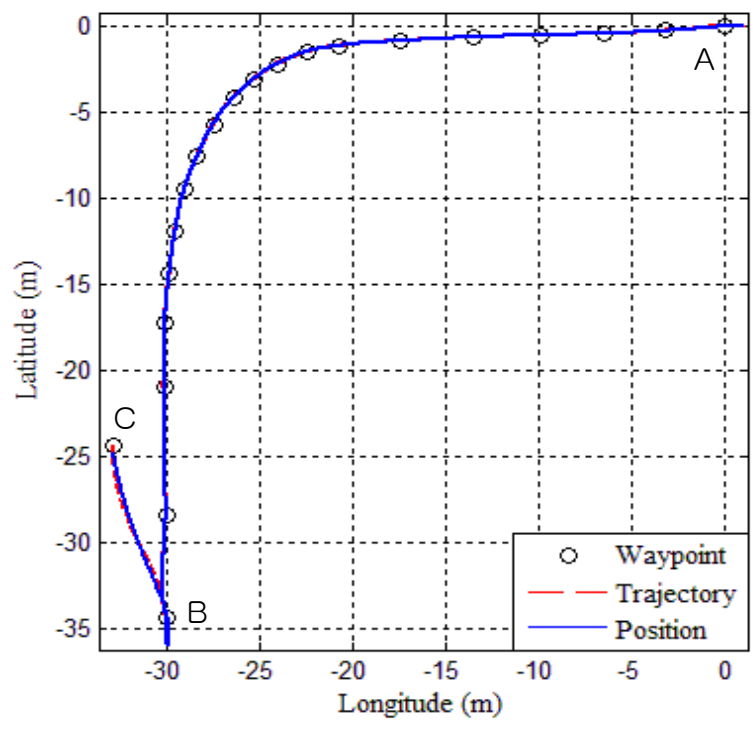

Figure 6. Waypoint, Traject ory, and Position of Vehicle
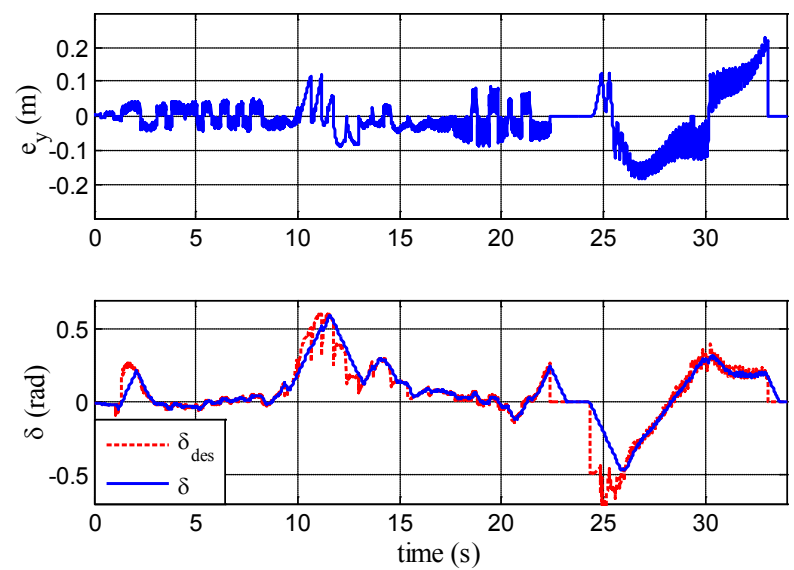

Figure 7. Time Responses of Lateral Error and Steering Angle without Consideration of Noise

Suppose measurement noise of position and yaw angle is considered with variation of $0.2(\mathrm{~m})$ and $0.5(\mathrm{deg})$ respectively. While the performance of the lateral controller in (10) is degraded with the maximum lateral error of about $0.5(\mathrm{~m})$ as shown in Fig. 9, one of the redesigned controller in (13) is not much in the presence of measurement noise and the kine mat ic constraints of the steering actuator (refer to Fig. 10). Fig. 11 shows the snapshot of forward following in the CarSim vehic le simu lator.
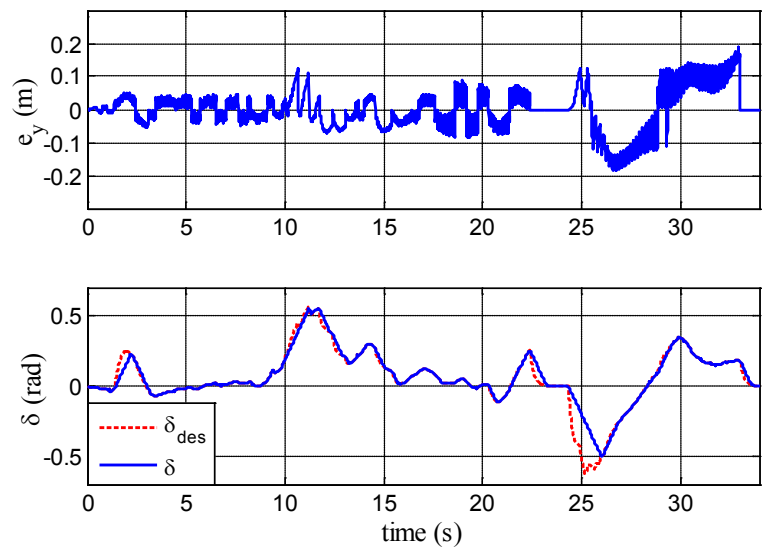

Figure 8. Time Responses of Lateral Error and Steering Angle of the Modified Lateral Controller
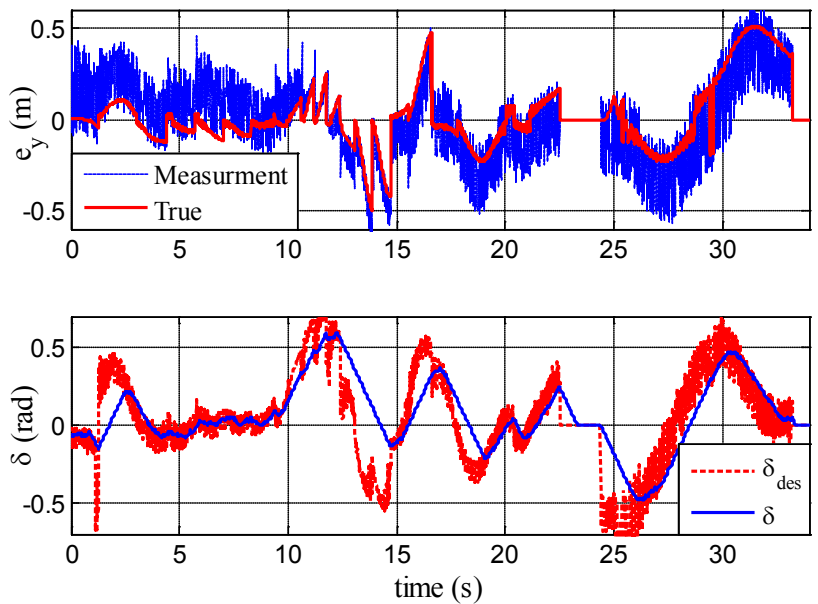

Figure 9. Time Responses of Lateral Error and Steering Angle with Consideration of Measurement Noise
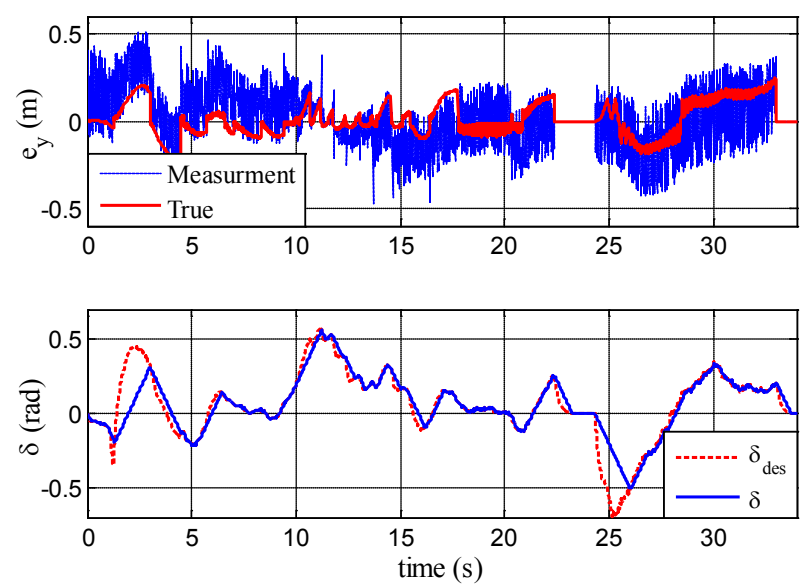

Figure 10. Time Responses of Lateral Error and Steering Angle of the Modified Lateral Controller 


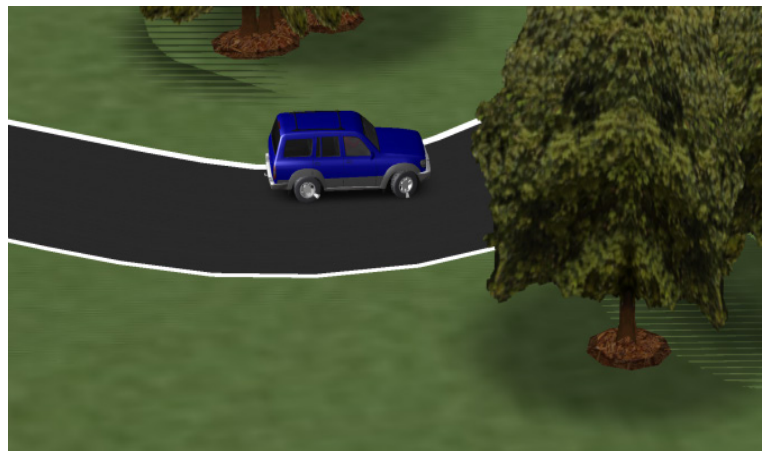

Figure 11. Snapshot of Vehicle Simulation for Forward Driving

The proposed lateral controller is implemented in a real vehicle as shown in Fig. 2 and its performance is evaluated via field driving tests. For the given 21 waypoints (see circle marks in Fig. 12), it is shown that the vehicle follows the desired trajectory with a maximum lateral error deviation of about $0.3(\mathrm{~m})$ (see also the second plot in Fig. 13). Furthermore, the corresponding time responses of the lateral controller are shown in Fig. 13. It is shown that the vehicle arrives at $20^{\text {th }}$ waypoint at about 65 second and waits until the gear is shifted to backward. Then, the parallel parking maneuver begins at about 78 second. Finally, snapshots of forward driving, temporary stop, and backward parallel parking are shown in Fig. 14.

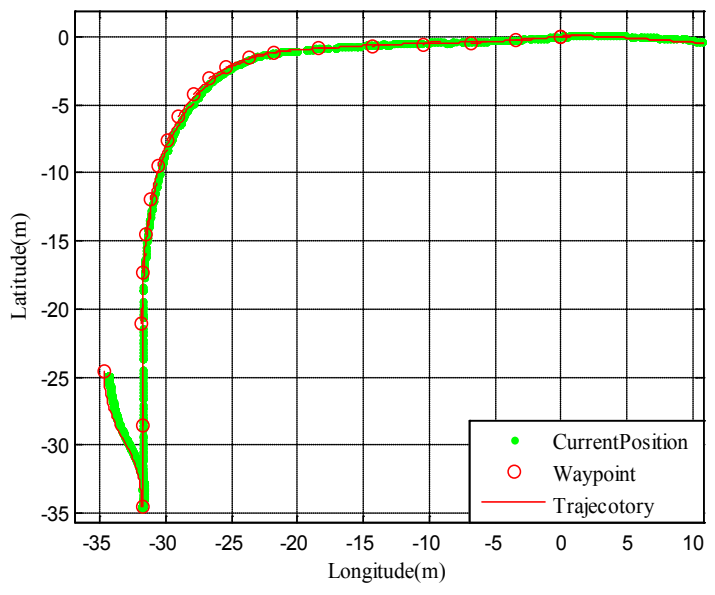

Figure 12. Position of Lat eral Controller in Field Test
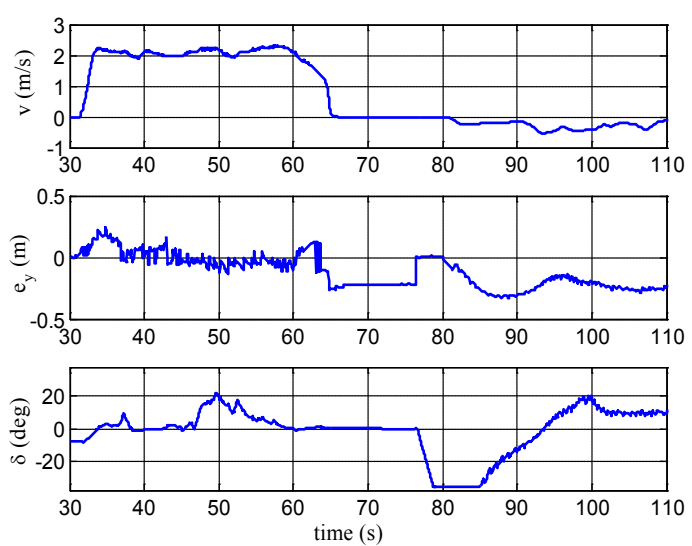

Figure 13. Time Responses of Lateral Controller in Field Test

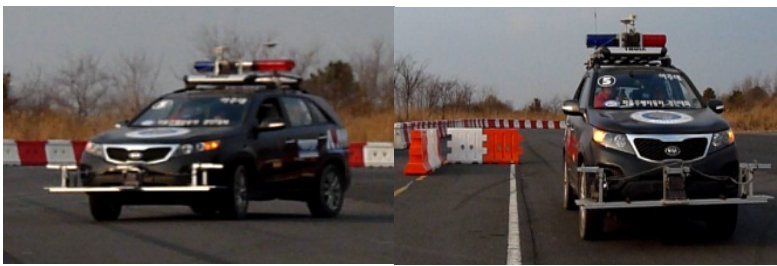

(a) Forward dri ving(b) Temporary stop for parking
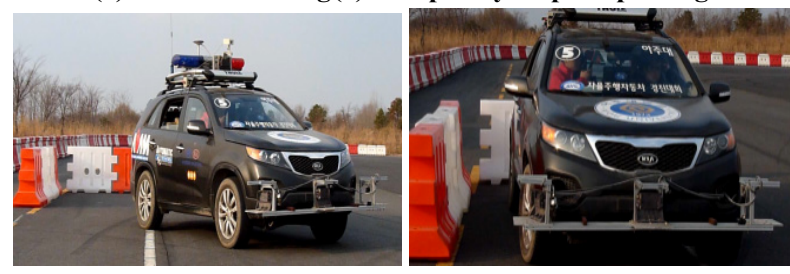

(c) Backward parking(d) Complete stop

Figure 14. Snapshots of Field Test

\section{Conclusions}

The lateral vehicle control algorith mbased on the vehicle model with kinematic constraints of the steering actuator was proposed. It was incorporated with the preview control, switching of multiple controllers, and filtering of the steering command to compensate for performance degradation due to measurement noise and manual control of velocity.Finally, it was validated via simu lations and field tests that the proposed controller could be applied to conduct two different driving maneuvers,i.e., forward driving and parallel backward parking.

In the near future, it is necessary to consider more various parking maneuvers such as perpendicular and/or multi-step parking, forward parking, and effect of time delay and packet loss when V2I communication is used.

\section{ACKNOWLEDGEMENTS}

This research was supported by the Industrial Strategic Technology Development Program (10035250, Development of Spatial Awareness and Autonomous Driving Technology for Automatic Valet Parking) funded by the Ministry of Knowledge Econo my (MKE, Korea).

\section{REFERENCES}

[1] Y. Kim , S. Hong, J. Kim, “Automatic guideline generation for parking assistance system based on on-screen display",The $13^{\text {th }}$ IEEE International Symposium on Consumer Electronics,pp. 845 - 846,2009.

[2] G. Yan, W. Yang, D. B. Rawat, and S. Olariu, "A secure and intelligent parking system", IEEE Intell.Trans. SystemsMagazine, vol. 3, no. 1, pp. 18-30, 2011.

[3] P. Jeevan, F. Harchut, B. M.-Bessler, and B. Huhnke, "Realizing autonomous valet parking with automotive grade 
sensors",Proc. of IEEE/RSJ Int. Conf. onIntell. Robots and Systems, Taipei,Taiwan, pp. 3824-3829, 2010.

[4] C. Baker and J. Dolan, "Street smarts for Boss",IEEE Robotics \& Automation Magazine, vol. 16, no. 1, pp. 78-87, 2009.

[5] G.Stanek, D. Langer, B. M.-Bessler, and B. Huhnke, "Junior 3: a test platform for advanced driver assistance systems", Proc. of IEEE Intell. Veh. Sym., San Diego, CA, USA, pp. 143-149, 2010.

[6] M. Chen and T. Chang, "A parking guidance and information system based on wireless sensor network", Proc. of IEEE Int. Conf. on Info. and Auto, pp. 601-605, 2011.

[7] K. Sung, J. Choi, and D. Kwak, "Vehicle control system for automatic valet parking with infrastructure sensors", Proc. of IEEE Int. Symp. on Cons. Elec, pp. 567-568., 2011.

[8] Y. K.Lo, A. B. Rad, C. W. L. Wong, and M. L.Ho, "Automatic parallel parking",Proc. of IEEE ITSC, pp. 531-540, 2003.

[9] K. Lee, D. Kim, W. Chung, H. W. Chang, P. Yoon, "Car parking control using a trajectory tracking controller", SICE-ICASE Int. Joint Conf., Busan, Korea, pp. 2058-2063,2006.
[10] B. Muller, J. Deutcher, and S. Grodde, "Continuous curvature trajectory design and feedforward control for parking a car", IEEE Trans. Cont. Sys. Tech., vol 15, no. 3, pp. 541-553, 2007.

[11] S. Fleury, P. Soueres, J.-P. Laumond, and R. Chatila, "Primitives for smoothing mobile robot trajectories", IEEE Trans. Robot. and Auto., vol. 11, no. 3, pp. 441-448, 1995.

[12] H. Peng and M. Tomizuka, "Preview control for vehicle lateral guidance in highway automation",ASME J. ofDynamicSy stems, Measurement, and Control, vol. 115, pp. 679-686, 1993.

[13] R. Rajamani, Vehicle Dynamics and Control, Springer,2006.

[14] R. Rajamani, C. Zhu, C. Chen, and L. Alexander, "Lateral control of a backward driven front-steering vehicle",ControlEng. Practice, vol. 11, pp. 531-540, 2003.

[15] B. Song and J. K. Hedrick, Dynamic surface control of uncertain nonlinear systems: an LMI approach, Springer,2011.

[16] CarSim, available at http://www.carsim.com 